\title{
Factors affecting sentinel lymph node metastasis in Turkish breast cancer patients: Predictive value of Ki-67 and the size of lymph node
}

\author{
Ozemir IA ${ }^{1}$, Orhun $\mathrm{K}^{1}$, Eren $\mathrm{T}^{1}$, Baysal $\mathrm{H}^{1}$, Sagiroglu $\mathrm{J}^{1}$, Leblebici $\mathrm{M}^{1}$, Ceyran $\mathrm{AB}^{2}$, Alimoglu $\mathrm{O}^{1}$ \\ Istanbul Medeniyet University, Goztepe Education and Research Hospital, Department of General Surgery, \\ Istanbul, Turkey.draliozemir@hotmail.com
}

\begin{abstract}
OBJECTIVES: We aimed to analyze the factors that affect the axillary lymph node involvement in Turkish breast cancer patients with clinically non-palpable axillary lymph node.

BACKGROUND: Sentinel lymph node biopsy is the gold standard technique to evaluate the axillary lymph node status that directly influences the prognosis and the treatment options in breast cancer.

METHODS: Breast cancer patients without axillary lymph node involvement in clinic examination were enrolled the study. Patients were categorized into the two groups according to existence of axillary lymph node metastasis or not. Demographic, histopathological and clinical data of patients were revealed retrospectively.

RESULTS: One-hundred and eighty-seven patients were analyzed and 101 of patients fulfilled the criteria and were included the study. Metastatic lymph node was detected in 38 (37.6\%) patients (Group 1), and was negative in $63(62.4 \%$ ) patients (Group 2). Sentinel lymph node metastasis were statistically significant higher in patients with Ki-67 $\geq 14 \%$ than patients with Ki-67<14\% $(51.9 \%$ vs $22.4 \%$; $p<0.01)$. Likewise, the mean size of the sentinel lymph node was statistically significant higher in Group 1 compared to Group $2(p<0.01)$. CONCLUSION: Ki-67 proliferation index and sentinel lymph node size may provide a higher prediction about the sentinel lymph node involvement in patients with clinically negative axillary lymph nodes (Tab. 3, Fig. 1, Ref. 31). Text in PDF www.elis.sk.

KEY WORDS: breast cancer, Ki-67, sentinel lymph node biopsy, lymph node size.
\end{abstract}

\section{Introduction}

Clinical and histopathological factors such as age, tumour size, tumour grade, estrogen receptor (ER) and progesterone receptor (PR) status, HER2 status and especially lymph node status directly influences the prognosis and the treatment option in patients with breast cancer (1). In the 1800 s, axillary lymph node dissection (ALND) was introduced as the standard surgical procedure for breast cancer and played an important role in patients' prognosis assessment and regional control of the disease (2). As the first site of tumour cell infiltration via lymphatic vessels, sentinel lymph node (SLN) is seen as a safety indicator to determine the spread of the disease at axillary region and to avoid the ALND in patients

${ }^{1}$ Istanbul Medeniyet University, Goztepe Education and Research Hospital, Department of General Surgery, Istanbul, Turkey, and ${ }^{2}$ Istanbul Medeniyet University, Goztepe Education and Research Hospital, Department of Pathology, Istanbul, Turkey

Address for correspondence: I.A. Ozemir, Istanbul Medeniyet University, Goztepe Education and Research Hospital, Department of General Surgery, Kucuksu Mah. Asma Sok., Eston Kandilli Evleri Sitesi, A/12 Blok, D:8, Usk Udar, Istanbul, Turkey.

Phone: +90.505.8032125, Fax: +90.216.3297224

Acknowledgement: This study was presented at 13. Turkish Breast Disease Congress 2015, Antalya, Turkey. with SLN-negative breast cancer (3). SLN biopsy has become the standard procedure in diagnostic management of patients with non-palpable axillary lymph nodes, since it was defined $(4,5)$. Histopathological evaluation of SLN can be performed using touch imprint cytology, frozen section (FS) analysis, or both. The reported sensitivity of frozen section analysis of SLN in literature ranges from 70-98\% (6). Axillary ultrasound examination reduces the false negativity of preoperative assessment at the level of 53 $\%$ compared to only clinical examination (3). On the other hand, $\mathrm{Ki}-67$ has a great potential as a prognostic and predictive factor in early breast cancer (7). Therefore, clinical, radiological and histopathological findings have a significant role to predict the SLN metastasis preoperatively. The study reported here analyzed the clinical, radiological and pathological data of 101 breast cancer patients with clinically negative axillary lymph nodes to reveal the risk factors for SLN metastasis.

\section{Methods}

\section{Patient selection}

This retrospective study was carried out in patients with breast cancer without axillary lymph node involvement at Istanbul Medeniyet University, Department of General Surgery, between April 2010 and September 2014. Patients with inflammatory 
cancers, pregnant patients, those who had axillary surgery, male patients, patients with multi-centric tumours and patients treated with neo-adjuvant chemotherapy and patients with unidentified sentinel node were excluded from the study. The informed consent was obtained from participants in this study.

\section{Sentinel lymph node biopsy}

Sentinel lymph node biopsy was performed through the methylene blue dye injection to the subareolar zone after general anesthesia. Eight minutes after the injection, $3 \mathrm{~cm}$ incision was made in the axillary region. Blue node was identified and excised with its lymphatic channel. Axillary region was examined for the presence of any other palpable rigid lymph node. Sentinel node was sent to the pathology laboratory for frozen section examination. Axillary dissection was performed in case of detection of metastasis in sentinel node. The surgeon did the appropriate breast surgery such as lumpectomy or mastectomy.

\section{Data analysis}

The patients were divided into the two groups according to the existence of sentinel lymph node metastasis in the definitive histopathology reports or not. Patients with metastasis in the sentinel lymph node were named as Group 1. Patients with the benign sentinel lymph node were named as Group 2. Patients' ultrasound reports and histopathological data including frozen section reports, tumour type, tumour size, and axillary lymph node status were

Tab. 1. Clinical and pathological characteristics of patients.

\begin{tabular}{|c|c|c|c|}
\hline & $\begin{array}{c}\operatorname{SLN}(+) \\
(n=38)\end{array}$ & $\begin{array}{l}\text { SLN (-) } \\
(n=63)\end{array}$ & $\mathrm{p}$ value \\
\hline Mean age (years) ${ }^{\dagger}$ & $54.3 \pm 12.1$ & $52.9 \pm 12.9$ & 0.575 \\
\hline \multicolumn{4}{|l|}{$\overline{\text { Age }}{ }^{\S}$} \\
\hline$-<40$ years $(n=15)$ & $4(10.5 \%)$ & $11(17.5 \%)$ & 0.342 \\
\hline$->40$ years $(n=86)$ & $34(89.5 \%)$ & $52(82.5 \%)$ & \\
\hline Tumour size $(\mathrm{cm})$ & $2.31 \pm 1.07$ & $1.92 \pm 0.98$ & 0.086 \\
\hline \multicolumn{4}{|l|}{ Tumour site ${ }^{\S}$} \\
\hline- left & $25(65.7 \%)$ & $28(44.4 \%)$ & $0.037 *$ \\
\hline - right & $13(34.3 \%)$ & $35(55.6 \%)$ & \\
\hline \multicolumn{4}{|l|}{ Histologic type ${ }^{\S}$} \\
\hline - invasive Ductal CA & $35(92.1 \%)$ & $44(69.8 \%)$ & $0.029 *$ \\
\hline - invasive Lobular CA & $3(7.9 \%)$ & $7(11.1 \%)$ & \\
\hline - invasive Mucinous CA & - & $3(4.8 \%)$ & \\
\hline \multicolumn{4}{|l|}{ SLN Frozen section ${ }^{\|, \S}$} \\
\hline - positive & $35(92.1 \%)$ & $2(3.2 \%)$ & $<0.01 * *$ \\
\hline - negative & $3(7.9 \%)$ & $61(96.8 \%)$ & r:0.894 \\
\hline \multicolumn{4}{|l|}{$\overline{\text { ER status }}$ s $^{\text {na }}$} \\
\hline - positive & $33(86.8 \%)$ & $53(84.1 \%)$ & 0.710 \\
\hline - negative & $5(13.2 \%)$ & $10(15.9 \%)$ & \\
\hline \multicolumn{4}{|l|}{$\overline{P R}$ status $^{\S}$} \\
\hline - positive & $33(86.8 \%)$ & $47(74.6 \%)$ & 0.142 \\
\hline - negative & $5(13.2 \%)$ & $16(25.4 \%)$ & \\
\hline \multicolumn{4}{|l|}{ HER-2 status $\S$} \\
\hline - Positive & $14(36.8 \%)$ & $26(41.3 \%)$ & 0.659 \\
\hline - Negative & $24(63.2 \%)$ & $37(58.7 \%)$ & \\
\hline \multicolumn{4}{|l|}{ ER percentage $\S$} \\
\hline-0 & $5(13.2 \%)$ & $10(15.9 \%)$ & 0.800 \\
\hline$-1-10 \%$ & $5(13.2 \%)$ & $6(9.5 \%)$ & \\
\hline$-67-100 \%$ & $18(47.3 \%)$ & $32(50.8 \%)$ & \\
\hline Allred Score $(0-8)($ Mean \pm SD) & $5.1 \pm 2.5$ & $5.2 \pm 2.7$ & 0.514 \\
\hline \multicolumn{4}{|l|}{ Molecular Subtypes ${ }^{\S}$} \\
\hline - luminal A/B group & $33(86.9 \%)$ & $53(74.2 \%)$ & 0.839 \\
\hline - HER-2 group & $2(5.3 \%)$ & $8(12.6 \%)$ & 0.079 \\
\hline - triple negative group & $3(7.8 \%)$ & $2(3.2 \%)$ & 0.823 \\
\hline Ki-67 percentage $($ Mean \pm SD) & $20.5 \pm 13.1$ & $16.4 \pm 18.8$ & $0.01 *$ \\
\hline \multicolumn{4}{|l|}{ Ki-67 Classification ${ }^{\S}$} \\
\hline$->14 \%$ & $27(71.1 \%)$ & $25(39.7 \%)$ & $0.002 * *$ \\
\hline$-<14 \%$ & $11(28.9 \%)$ & $38(60.3 \%)$ & \\
\hline
\end{tabular}

SLN - Sentinel lymph node, ER - Estrogen receptor, PR - Progesterone receptor, $r$ - Spearman's rho correlation value, $\$-$ Mann-Whitney Utest, $\S-$ Pearson Chi-Square, $\dagger-$ T-Test, " - Sperman's rho correlation, ${ }^{*}$ - significant at the 0.05 level, $*^{*}$ - significant at the 0.01 level. 
reviewed retrospectively. Immunohistochemical (IHC) staining was performed for ER, PR, HER-2 and Ki-67 for each patient. The Allred scoring system was used to evaluate ER status (8). The percentage and intensity scores were revealed, and tumour cells with a total score of 3-8 were considered ER and PR positive. Scores of 0 and 1 were considered negative for HER-2 expression according to IHC and score 3 was considered as positive. In case of HER-2 score 2, FISH analysis were performed. While assessing the Ki-67 expression, values $>14 \%$ were considered as positive as suggested by St. Gallen $(4,9)$. The mean tumour size, mean size of sentinel lymph node and number of total metastatic lymph nodes was compared according to Ki-67 proliferation index of patients. We also performed subtype classification by IHC based on ER, PR and HER-2 status and Ki-67 expression.

SPSS software version 20.0 (SPSS Inc., Chicago, IL) was used to analyze the data of this study. Descriptive statistics was applied in relevant parameters. Distributions of the numerical variables were examined by histograms and Shapiro-Wilk test. Where appropriate, comparisons of categorical variables were performed using the chi-squared test and continuous variables with median or mean values were compared using the Mann-Whitney U test. Spearman's rho correlation coefficient was used for the correlation analysis between the parameters. The results were reviewed in the confidence interval of $95 \%$, and the value $\mathrm{p}<0.05$ was considered statistically significant.

This study was approved by the Scientific Ethics Committee of the Istanbul Medeniyet University, School of Medicine.

\section{Results}

Totally, 187 breast cancer patients underwent surgical treatment in this process. Clinically negative axillary lymph node was detected in 101 of the 187 patients and included in the study to evaluate the risk and predictive factors for SLN metastasis. The mean age of the patients was $53.4 \pm 12.6$ years and the mean tumour size was $2.07 \pm 1.03 \mathrm{~cm}$. In $38(37.6 \%)$ patients, the SLN metastasis was shown in the final pathological examination and so underwent axillary lymph node dissection (Group 1). SLN biopsy was detected benign in $63(62.4 \%)$ patients (Group 2). Range of harvested SLN was 1-4 lymph nodes in each surgery (mean; $1.51 \pm 0.72$ ).

The mean age of patients in Group 1 was $54.3 \pm 12.1$ years, while those in Group 2 was $52.9 \pm 12.9$ years $(p=0.575)$. We subcategorized the patients in terms of younger and older than 40 years. Four (10.5\%) of the patients in Group 1 and 11 (17.5 $\%$ ) of the patients in Group 2 were younger than 40 years ( $\mathrm{p}=$ 0.342 ). The mean tumour sizes of Group 1 and Group 2 were 2.31 $\pm 1.07 \mathrm{~cm}$ and $1.92 \pm 0.98 \mathrm{~cm}$, respectively $(\mathrm{p}=0.086)$. Univariate analysis showed that there were no significant differences between Group 1 and Group 2 according to demographic data like age and tumour size (Tab. 1). Likewise, there was no significant difference between the groups according to IHC staining features, such as ER, PR and HER-2 receptor status, Allred scoring system, ER staining percentages and molecular subtypes (Tab. 1).

In fifty-three $(52.5 \%)$ of the patients, the tumour was on the left breast, and in $48(47.5 \%)$ on the right breast. Twenty-five (65.7\%) of the left side tumours and $13(34.3 \%)$ of the right side tumours had SLN metastasis. SLN metastasis were detected statistically significantly higher on the left side tumours than right side tumours $(p=0.037)$. SLN metastasis were detected statistically significantly higher in patients with invasive ductal carcinoma than other histologic types $(p=0.029)$. The mean size of the sentinel lymph node in Group 1 was statistically significantly higher than in Group 2 $(1.57 \pm 0.75 \mathrm{~cm}$ versus $1.35 \pm 0.64 \mathrm{~cm}$, respectively, $\mathrm{p}=0.044)$. The mean Ki-67 proliferation index of patients in Group 1 was detected $20.5 \pm 13.1$, while it was detected $16.4 \pm 18.8$ in Group 2. The mean Ki-67 value was statistically significantly higher in Group 1 than in Group $2(\mathrm{p}=0.01)$ (Tab. 1).

According to the suggestion of St Gallen, patients were subcategorized in terms of Ki-67 values that were higher or lower than $14 \%$. Twenty-seven $(71.1 \%)$ of the patients in Group 1 had $>14$ $\% \mathrm{Ki}-67$ expression while it was $25(39.7 \%)$ in Group 2. SLN metastasis was detected statistically significant higher in patients with $>14 \% \mathrm{Ki}-67(\mathrm{p}=0.002)$. The mean tumour size was determined statistically significantly higher in patients with $>14 \%$ Ki-67 proliferation index $(2.26 \pm 0.90 \mathrm{~cm}$ versus $1.86 \pm 1.12 \mathrm{~cm}$, $\mathrm{p}=0.01)$. Number of metastatic lymph nodes was compared and significant difference was detected between Ki-67 subgroups. The mean metastatic lymph node number was $2.15 \pm 5.04$ in patients with $>14 \% \mathrm{Ki}-67$ proliferation index, while it was $0.51 \pm 1.50$ in patients with $<14 \% \mathrm{Ki}-67$ proliferation index $(\mathrm{p}=0.004)$. Ki-67 values higher than $14 \%$ were statistically significant and positively correlated with SLN positivity $(\mathrm{r}=0.258)$, tumour size $(0.222)$ and number of metastatic lymph nodes $(r=0.224)$ (Tab. 2, Fig. 1).

We analyzed the sensitivity, specificity, positive predictive value (PPV) and negative predictive values (NPV) of the ultra-

Tab. 2. Tumour and axillary lymph node features according to Ki-67 subgroups.

\begin{tabular}{|c|c|c|c|c|}
\hline & $\begin{array}{c}\text { Ki-67 }>\% 14 \\
\text { (n:52) }\end{array}$ & $\begin{array}{c}\text { Ki-67 }<\% 14 \\
\text { (n:49) }\end{array}$ & $\mathrm{p}$ value & r value \\
\hline SLN size† & $1.59 \pm 0.79$ & $1.27 \pm .53$ & 0.079 & - \\
\hline Tumour size $^{\dagger}$ & $2.26 \pm 0.90$ & $1.86 \pm 1.12$ & $0.01 * *$ & $0.222 *$ \\
\hline \multicolumn{5}{|l|}{$\mathrm{SLN}^{*}$} \\
\hline - Positive & $27(71.1 \%)$ & $11(28.9 \%)$ & $0.002 * *$ & $0.258 *$ \\
\hline - Negative & $25(39.7 \%)$ & $38(60.3 \%)$ & & \\
\hline Number of Metastatic Lymph Nodes ${ }^{\dagger}$ & $2.15 \pm 5.04$ & $0.51 \pm 1.50$ & $0.004 * *$ & $0.224 * *$ \\
\hline
\end{tabular}



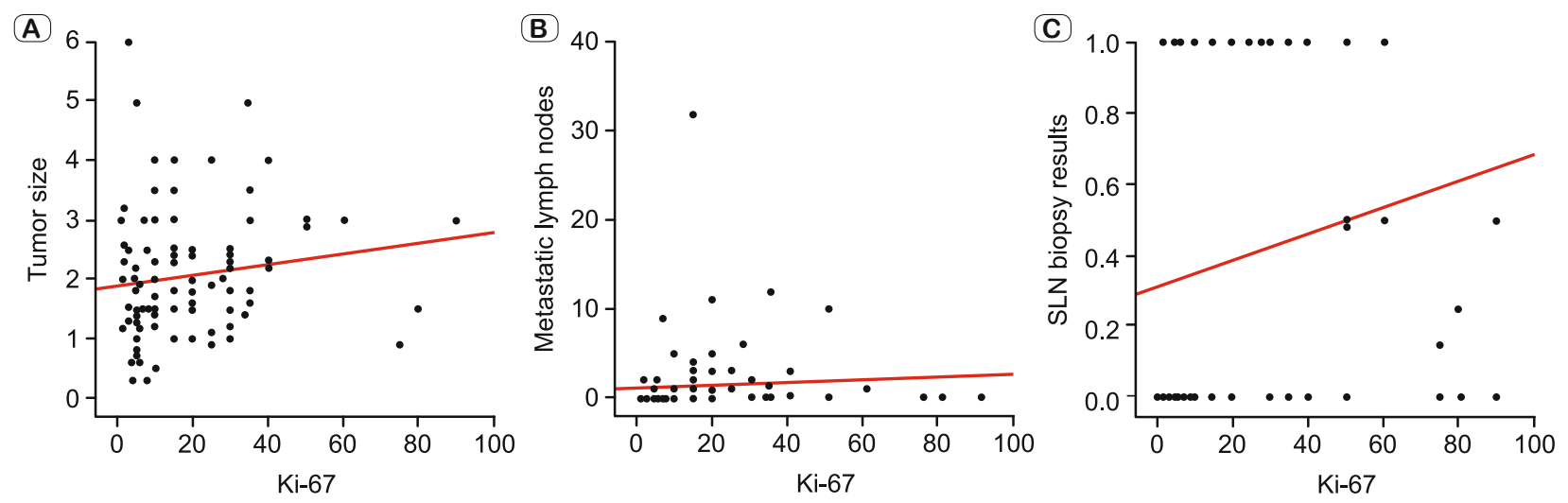

Fig. 1. Scatter-plot graphics; A - correlation between Ki-67 values and tumour size, B - correlation between Ki-67 values and number of metastatic lymph nodes, $\mathrm{C}$ - correlation between Ki-67 values and SLN biopsy results

sonography (USG), frozen section (FS), magnetic resonance imaging (MRI) and Ki-67 expression percentages to compare the diagnostic significance of these methods to detect the metastatic axillary lymph node in this specific patient group (Tab. 3). We revealed that FS had the highest diagnostic significance among these methods. Statistical analysis showed FS results had a statistically significant and positive correlation relationship $(r=0.894)$ with definitive histology reports of SLN (Tab. 1). We determined that Ki-67 expression ratio had similar predictive significance with USG and MRI examination in terms of detecting SLN metastasis in patients with clinically negative axilla.

\section{Discussion}

SLN biopsy has provided a decreased morbidity and it has become the standard of care for breast cancer management (10). It continues to be an issue of an extensive study in the management of breast cancer since it was first defined in 1997 (11). SLN metastasis rate is about $30-35 \%$ in patients with early stage breast cancer (12), and it was reported that $40-70 \%$ of patients with metastatic SLN were free of metastasis in non-SLNs (13). Therefore, prediction of SLN status preoperatively with biomarkers or histopathological features of the tumour may allow us to avoid the unnecessary SLN biopsies in selected patients. Nomogram is a predictive model used to identify sentinel or additional lymph node metastasis at several facilities. Nomograms and other scoring systems are based on patients' clinicopathological data and performed preoperatively $(14,15)$. In this study we analyzed the factors that were related to patients and tumour characteristics, and their predictive or causing effects on the SLN metastasis.

ALND and SLN biopsy provide postoperative staging of the disease, therefore preoperative diagnostic modalities have significance to assess the axilla in breast cancer patients, especially in patients being prepared for SNL biopsy. Nowikiewicz (3) et al revealed that USG scan is the most available, the cheapest, and the repeatable method if performed in the period directly preceding SLN biopsy. If performed more than 4 weeks before surgery, false negative results increased and diagnostic significance decreased. We found the false negative results in 10/44 (22.7\%) patients. Various studies showed that USG, computed tomography and MRI have sensitivities $72-78 \%, 78 \%$ and $67 \%$ and specificities $77-78$ $\%, 75 \%$ and $78 \%$, respectively to detect the metastatic lymph node in breast cancer patients (16). Flurodeoxyglucose positron emission tomography (PET/CT) was evaluated in various studies

Tab. 3. Sensitivity, specificity, PPV and NPV of the diagnostic methods in terms of detecting the metastasis of SLN in patients with clinically negative axilla.

\begin{tabular}{|c|c|c|c|c|c|c|}
\hline & Group 1 & Group 2 & Sensitivity & Specificity & PPV & NPV \\
\hline \multicolumn{7}{|l|}{ USG } \\
\hline - suspicious lymph metastasis & 29 & 28 & \multirow{2}{*}{$76.3 \%$} & \multirow{2}{*}{$55.5 \%$} & \multirow{2}{*}{$50.9 \%$} & \multirow{2}{*}{$79.5 \%$} \\
\hline - benign lymph nodes & 9 & 35 & & & & \\
\hline \multicolumn{7}{|l|}{ MRI } \\
\hline - suspicious lymph metastasis & 16 & 23 & \multirow{2}{*}{$72 \%$} & \multirow{2}{*}{$50 \%$} & \multirow{2}{*}{$41 \%$} & \multirow{2}{*}{$71.9 \%$} \\
\hline - benign lymph nodes & 9 & 23 & & & & \\
\hline \multicolumn{7}{|l|}{ Frozen section } \\
\hline - malign SLN & 35 & 2 & \multirow{2}{*}{$92.1 \%$} & \multirow{2}{*}{$96.8 \%$} & \multirow{2}{*}{$94.6 \%$} & \multirow{2}{*}{$95.3 \%$} \\
\hline - benign SLN & 3 & 61 & & & & \\
\hline \multicolumn{7}{|l|}{ Ki-67 expression } \\
\hline$->14 \%$ & 27 & 25 & \multirow{2}{*}{$71.1 \%$} & \multirow{2}{*}{$60.3 \%$} & \multirow{2}{*}{$51.9 \%$} & \multirow{2}{*}{$77.6 \%$} \\
\hline$-<14 \%$ & 11 & 38 & & & & \\
\hline
\end{tabular}

USG - Ultrasonography, MRI - Magnetic resonance imaging, PPV - Positive predictive value, NPV - Negative predictive value, SLN - Sentinel lymph node 
for detecting the axillar metastasis. It was revealed less sensitive (20-30\%) but more specific (>95\%) than other modalities (17). In this study, USG and MRI sensitivity was similar to literature ( $76.3 \%$ and $72 \%$, respectively), but specificities were lower than literature (55.5\% and $50 \%$, respectively).

Morrison (18) et al revealed that young patients demonstrated more aggressive features and worse outcomes when compared to older patients, and Han (19) et al supported that young age was an independent risk factor for relapse. We analyzed and compared the mean ages and subgroups of young $(<40$ years) and older $(>40$ years) patients according to SLN metastasis, significant difference was not detected between Group 1 and Group 2.

There is no data available in literature that SLN metastasis is more common in left or right breast cancers. But in this study, we revealed a statistically significantly higher SLN metastasis in left side breast cancers $(\mathrm{p}<0.05)$.

$\mathrm{Wu}(20)$ et al reported in their studies including 1325 patients, that the mean tumour size was $2.9 \mathrm{~cm}$ in SLN positive group, while it was 2.1 in SLN negative group. They revealed a statistically significant difference between the groups. In the present study, the mean tumour size of Group 1 was larger than in Group 2 without a statistically significance $(\mathrm{p}>0.05)$.

HER-2-positive and triple negative breast cancers are more likely to exhibit higher metastatic lymph node involvement and worse survival, compared to luminal-like (luminal-A and luminalB) tumours (21). Kumar (22) et al revealed that triple negative breast cancers are associated with younger age, higher grade and advanced stage at diagnosis. Dunnwald (23) et al compared ER+/ $\mathrm{PR}+, \mathrm{ER}+/ \mathrm{PR}-, \mathrm{ER}-/ \mathrm{PR}+$ and ER-/PR- breast cancer patients, and they found that SLN metastasis was statistically significantly higher in ER-/PR+ and ER-/PR- patients than in others. In the study by Crawford (24) et al, the authors reported the hormone receptor status of breast cancer patients. According to this study, $83.5 \%$ of ER, $76.4 \%$ of PR, and $34.4 \%$ of HER- 2 were positive in patients with SLN metastasis. Ratios were 83.0 \%, 78.5\% and $21.8 \%$, respectively, in patients with negative SLN biopsy. They found statistically significantly higher HER-2 positivity in SLN positive group. We did not find significant difference between the groups according to molecular subtype categories or hormone receptor status in patients with clinical negative axilla.

Allred scoring system is a clinical instrument based on the percentage of cells that stain by IHC for ER (on a scale of 0 to 5) and the intensity of that staining (on a scale of 0 to 3 , for a possible total score of 8 ). Mohammed (25) et al revealed that patients with higher Allred score have longer disease free survival. Water (26) et al found the similar results that higher Allred scores related with better survival rates. We compared the Allred scores according to existence of metastasis in SLN biopsy. There was no significant difference between the patients in Group 1 and Group 2 .

Ki-67 was found to be universally expressed among proliferating cells and absent in quiescent cells, making it ripe for evaluation as a tumour proliferation biomarker (27). It is well documented that tumour cell proliferation by $\mathrm{Ki}-67$ expression is strongly associated with breast cancer prognosis (7). Inic (28) et al considered the cut-off at $14 \%$ for Ki-67, and they showed that higher Ki-67 values were related with the axillary lymph node involvement. Likewise, Matsonneuve (29) et al determined that higher Ki-67 values than $14 \%$ were related to worse prognosis, axillary and distant metastasis. In our study, we revealed statistically significantly higher Ki-67 expression values in patients with metastatic SLN than in patients with benign SLN. Shokouh (30) et al. revealed that higher Ki-67 index tumours showed more HER-2 overexpression, larger size and more lymph node involvement. Also, we have performed the subgroup analysis in terms of Ki-67 proliferation index value. Higher Ki-67 values were statistically significantly related and positively correlated with larger tumour size and increased number of metastatic lymph nodes. Recent studies and some of the guidelines suggested omitting the axillary dissection in selective patients even if SLN biopsy was positive (31). Ki-67 proliferation index ratio and size of SLN can play an important role on decision-making to perform axillary dissection or not.

This study showed that larger size of the sentinel lymph node and higher Ki-67 values, especially $>14 \%$ were related with metastatic SLN. We thought that addition of these criteria to USG examination might provide a higher prediction about the axillary lymph node involvement in patients with clinically negative axilla. Also, correlation between higher Ki-67 values and non-sentinel lymph node involvement is an important factor in the clinical management of this patient group.

\section{Learning points}

1) Ki-67 proliferation index higher than $14 \%$ is an important cutoff point for Turkish breast cancer patients, likewise presented in some international studies.

2) The larger size of SLN and Ki-67 proliferation index $>14 \%$ may be provide the prediction of existence of metastatic SLN and increased number of metastatic lymph nodes in breast cancer.

\section{Reference}

1. Wishart GC, Rahha E, Green A, Ellis I, Ali HR, Provenzano E et al. Inclusion of KI67 significantly improves performance of the PREDICT prognostication and prediction model for early breast cancer. BMC Cancer 2014; 14: 908.

2. Jatoi I, Hilsenbeck SG, Clark GM, Osborne CK. Significance of axillary lymph node metastasis in primary breast cancer. J ClinOncol 1999; 17: 2334-2340.

3. Nowikiewicz T, Nowak A, Wisniewska M, Wisniewska M, Zegarski W. Diagnostic value of preoperative axillary lymph node ultrasound assessment in patients with breast cancer qualified for sentinel lymph node biopsy. Videosurg Miniinv 2015; 10: 170-177.

4. GoldhirschA, Wood WC, Coates AS, Gelber RD, Thürlimann B, Senn HJ et al. Strategies for subtypes: dealing with the diversity of breast cancer: highlights of the St. Gallen International Expert Consensus on the Primary Therapy of Early Breast Cancer 2011. Ann Oncol 2011; 22: 1736-1747.

5. Krag DN, Anderson SJ, Julian TB, Brown AM, Harlow SP, Costantino JP et al. Sentinel-lymph-node resection compared with conventional axillary-lymph-node dissection in clinically node-negative patients with breast cancer: overall survival findings from the NSABP B-32 randomised phase 3 trial. Lancet Oncol 2010; 11: 927-933. 
6. Vohra LM, Gulzar R, Saleem O. Intraoperative frozen examination of sentinel lymph node in breast cancer. J Ayub Med Coll Abbottabad 2015; 27: 40-44.

7. Yerushalmi R, Woods R, Ravdin PM, Hayes MM, Gelmon KA. Ki67 in breast cancer: prognostic and predictive potential. Lancet Oncol 2010; 11: 174-183.

8. Allred DC, Harvey JM, Berardo M, Clark GM. Prognostic and predictive factors in breast cancer by immunohistochemical analysis. Mod Pathol 1998; 11: 155-168.

9. De Azambuja E, Cardoso F, De Castro G, Jr, Colozza M, Mano MS, Durbecq V et al. Ki-67 as prognostic marker in early breast cancer: a meta-analyis of published studies involving 12,155 patients. Br J Cancer 2007; 96: 1504-1513.

10. Dabakuyo TS, Fraisse J, Causeret S, Gouy S, Padeano MM, Loustalot $\mathrm{C}$ et al. A multicenter cohort study to compare quality of life in breast cancer patients according to sentinel lymph node biopsy or axillary lymph node dissection. Ann Oncol 2009; 20: 1352-1361.

11. Giuliano AH, Jones RC, Brennan M, Statman R. Sentinel lymphadeneetomy in breast cancer. J Clin Oncol 1997; 15: 2345-2350.

12. Bland KI, Menck HR, Scott-Conner CE, Morrow M, Winchester DJ, Winchester DP. The National Cancer Data Base 10-year survey of breast cancer treatment at hospitals in the United States. Cancer 1998; 83: $1262-1273$.

13. Abdessalam SF, Zervos EE, Prasad M, Farrar WB, Yee LD, Walker MJ et al. Predictors of positive axillary lymph nodes after sentinel lymph node biopsy in breast cancer. Am J Surg 2001; 182: 316-320.

14. Barranger E, Coutant C, Flahault A, Delpech Y, Darai E, Uzan S. An axilla scoring system to predict non-sentinel lymph node status in breast cancer patients with sentinel lymph node involvement. Breast Cancer Res Treat 2005; 91: 113-119.

15. Pal A, Provenzano E, Duffy SW, Pinder SE, Purushotham AD. A model for predicting non-sentinel lymph node metastatic disease when the sentinel lymph node is positive. Br J Surg 2008; 95: 302-309.

16. Riegger C, Koeninger A, Hartung V, Otterbach F, Kimmig R, Forsting $\mathbf{M}$ et al. Comparison of the diagnostic value of FDG-PET/CT and axillary ultrasound for the detection of lymph node metastases in breast cancer patients. Acta Radiol 2012; 53: 1092-1098.

17. Pritchard KI, Julian JA, Holloway CMB, McCready D, Gulenchyn KY, George R et al. Prospective study of 2-( $\left(^{18} \mathrm{~F}\right)$ Fluorodeoxyglucose positron emission tomography in the assessment of regional nodal spread of disease in patients with breast cancer: An Ontario clinical oncology group study. J Clin Oncol 2012; 30: 1274-1279.

18. Morrison DH, Rahardja D, King E, Peng Y, Sarode VR. Tumour biomarker expression relative to age and molecular subtypes of invasive breast cancer. Br J Cancer 2012; 107: 382-387.

19. Han W, Kim SW, Park IA, Kang D, Kim SW, Youn YK et al. Young age: an independent risk factor for disease-free survival in women with operable breast cancer. BMC Cancer 2004; 4: 82.
20. Wu JL, Tseng HS, Yang LH, Wu HK, Kuo SJ, Chen ST et al. Prediction of axillary lymph node metastases in breast cancer patients based on pathologic information of the primary tumour. Med Sci Monitor: Intern Med J Exp Clin Res 2014; 20: 577.

21. Yu KD, Jiang YZ, Shao ZM. Difference between observed and expected number of involved lymph nodes reflects the metastatic potential of breast cancer independent to intrinsic subtype. Oncotarget 2015; 30 (6): 16686-16697.

22. Kumar P, Aggarwal R. An overview of triple negative breast cancer. Arch Gynecol Obstet 2015 (epubhead of print).

23. Dunnwald LK, Rossing MA, Li CI. Hormone receptor status, tumour characteristics, and prognosis: a prospective cohort of breast cancer patients. Breast Cancer Res 2007; 9 (1): R6.

24. Crawford JD, Ansteth M, Barnett J, Glissmeyer M, Johnson NG. Routine completion axillary lymph node dissection for positive sentinel nodes in patients undergoing mastectomy is not associated with improved local control. Amer J Surg 2013; 205: 581-584.

25. Mohammed Z, Edwards J, Orange C, Mallon E, Doughty JC, McMillan DC et al. Breast cancer outcomes by steroid hormone receptor status assessed visually and by computer image analysis. Histopathol 2012; 61: 283-292.

26. van de Water W, Fontein DB, van Nes JG, Bartlett JM, Hille ET, Putter $\mathbf{H}$ et al. Influence of semi-quantitative oestrogen receptor expression on adjuvant endocrine therapy efficacy in ductal and lobular breast cancer - a TEAM study analysis. Eur J Cancer 2013; 49: 297-304.

27. Lopez F, Belloc F, Lacombe F, Dumain P, Reiffers J, Bernard P et al. Modalities of synthesis of Ki67 antigen during the stimulation of lymphocytes. Cytometry 1991; 12: 42-49.

28. Inic Z, Zegarac M, Inic M, Markovic I, Kozomara Z, Djurisic I et al. Difference between Luminal A and Luminal B Subtypes According to Ki-67, Tumour Size, and Progesterone Receptor Negativity Providing Prognostic Information. Clin Med Insights Oncol 2014; 8: 107.

29. Maisonneuve P, Disalvatore D, Rotmensz N, Curigliano G, Colleoni M, Dellapasqua S et al. Proposed new clinicopathological surrogate definitions of luminal A and luminal B (HER2-negative) intrinsic breast cancer subtypes. Breast Cancer Res 2014; 16: R65.

30. Shokouh TZ, Ezatollah A, Barand P. Interrelationships Between Ki67, HER2/neu, p53, ER, and PR Status and Their Associations With Tumour Grade and Lymph Node Involvement in Breast Carcinoma Subtypes: Retrospective-Observational Analytical Study. Medicine 2015; 94: e1359.

31. Giulliano AE, McCall LM, Beitsch PD, Whitworth PW, Morrow M, Blumencranz PW et al. ACOZOG Z0011: A randomized trial of axillary node dissection in women with clinical T1-2 N0 M0 breast cancer who have a positive sentinel node. J Clin Oncol 2010; 28: 18s.

Received February 6, 2016. Accepted February 17, 2016. 